\title{
Transition to turbulence in an elliptic vortex
}

\author{
By T. S. Lundgren ${ }^{1}$ AND N. N. Mansour
}

\section{Motivation and objectives}

We study the three dimensional instability and nonlinear growth of the twodimensional flow described by the streamfunction

$$
\Psi=\frac{A \sin b_{1} x \sin b_{2} y}{b_{1}^{2}+b_{2}^{2}}
$$

where $b_{1}=\pi / L_{1}, b_{2}=\pi / L_{2}$. This is a swirling flow in a box which is bounded by $0 \leq x \leq L_{1}, 0 \leq y \leq L_{2}$ and is infinite in the $z$ direction. This flow is a solution of the Navier-Stokes equation with $A=\exp \left(-\nu\left(b_{1}^{2}+b_{2}^{2}\right) t\right)$ which slowly decays. We seek a viscous solution which starts near this one and slips along but does not penetrate the bounding walls. The vorticity of the basic flow is $\omega_{z}=A \sin b_{1} x \sin b_{2} y$ which has maximum value $A$ at the center of the box and drops to zero at the boundaries. We can think of the resulting flow as that of a captive vortex.

Denote $E=L_{1} / L_{2}$. What is interesting about this flow is that when $E \neq 1$, it is unstable to three-dimensional disturbances. Periodic waves grow along the $z$ direction, causing the captive vortex to distort into a snake-like configuration. Components of vorticity perpendicular to the $z$ axis grow. As the instability grows, we find that the vorticity becomes increasingly sheet-like. Ultimately, the sheets break up into a turbulent mixture of intense vortex tubes. When $E=1$, the flow is completely stable.

The streamline pattern of the basic flow is elliptical in the central part of the box, with aspect ratio $E$, becoming more rectangular as the boundaries are approached. Figure 1 shows the streamline pattern when $E=2$. Bayly (1989) has shown analytically and numerically that the flow is unstable to high wavenumber disturbances when the Reynolds number is large. At high wavenumber, the instability in this bounded geometry is similar to the broad band instability in an unbounded flow with elliptical streamlines and uniform vorticity studied by Pierrehumbert (1986), Bayly (1986), Waleffe $(1989,1990)$, and Landman and Saffman (1987). The mechanism for the instability is a resonance between inertial oscillations, which can exist when the streamlines are circular, and the periodicity introduced by the ellipticity.

The work which motivated these studies was that of Orszag and Patera (1983). They numerically found a three-dimensional instability on a flow which is a superposition of a Tollmein-Schlichting wave and the Blasius boundary layer flow. This flow contains an elongated elliptically shaped swirling region. It is believed that the 


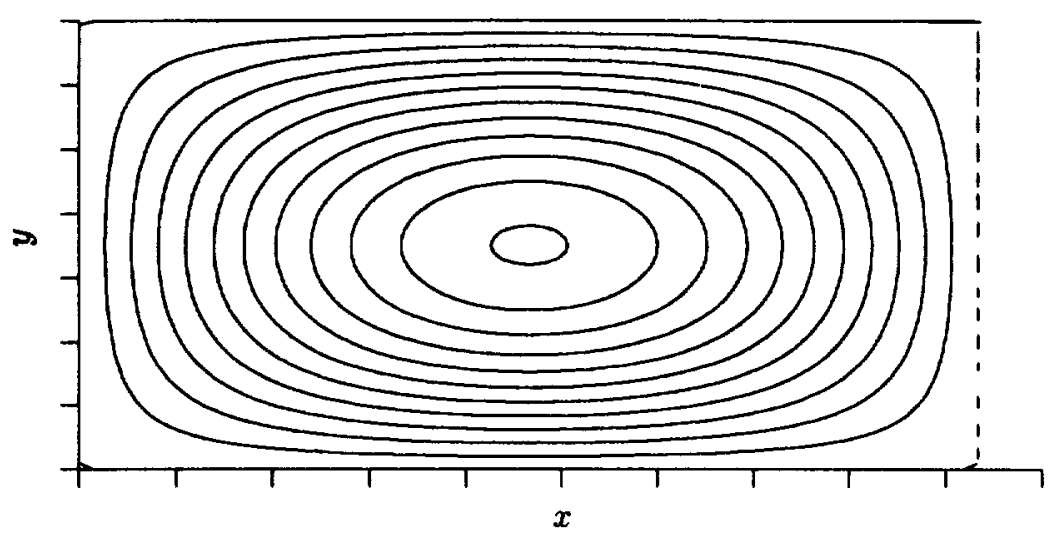

FIgURE 1. Streamline pattern and vorticity contours for $E=2$. The tick marks are at .6 intervals in the $x$-direction and .4 intervals in the $y$-direction.

wavy instability which develops explains the generation of streamwise vorticity in boundary layer transition.

An even earlier work of Gledzer et al. $(1974,1975)$ showed instability in a swirling flow within an elliptic cylinder. The experiment consisted of rotating a water-filled elliptic cylinder until solid body rotation was achieved. Upon stopping the rotation, the water continues to rotate with approximately elliptical streamlines. It was observed that the flow rapidly developed swirls perpendicular to the rotation axis, with one or more cells depending on the length of the cylinder. Malkus (1989) did clever experiments with a water-filled flexible cylinder which was made elliptical by rotating it between stationary rollers. In this flow, the unstable wave rapidly flashes into small scale turbulence.

There is another class of flows which is closely related to the present work. This is the bending instability of a sharp-edged vortex with uniform vorticity exposed to a transverse straining flow treated by Widnall, Bliss and Tsai (1974), Moore and Saffman (1975), and Robinson and Saffman (1984). In this flow, wavy disturbances, comparable in length to the dimensions of the vortex, grow on the vortex. In the absence of the straining flow, non-rotating planar waves exist. The strain causes such a wave to align with and grow along the plane of maximum strain rate. In our flow, the vorticity distribution rises smoothly to a maximum at the center instead of being sharp-edged, and there are finite boundaries; nevertheless, some of the stability results are very similar.

In the present work, we show numerically that the basic flow described by Eq. (1) is unstable at modest axial wavenumbers when the Reynolds number is greater than a critical value. In section 2 , we present stability diagrams which superficially resemble those for two-dimensional parallel flows. In section 3 , we present the results of a nonlinear computation which clearly shows transition to turbulent flow.

\section{Stability}

For the stability problem, we set $A=1$, neglecting the slow decay. This is similar 
to the traditional method of studying the stability of a growing boundary layer by treating it as a parallel flow. In the usual way, we decompose the velocity field into the velocity field $\mathbf{U}$ of the basic flow plus a small perturbation $\mathbf{u}^{\prime}$. The linearized equations for $\mathbf{u}^{\prime}$ are

$$
\begin{gathered}
\nabla \cdot \mathbf{u}^{\prime}=\mathbf{0} \\
\frac{\partial \mathbf{u}^{\prime}}{\partial t}+\nabla \cdot\left(\mathbf{U} \mathbf{u}^{\prime}+\mathbf{u}^{\prime} \mathbf{U}\right)=-\nabla p^{\prime}+\nu \nabla^{2} \mathbf{u}^{\prime} .
\end{gathered}
$$

We separate variables in the form

$$
\begin{aligned}
& u^{\prime}=\exp i k_{z} z \frac{1}{b_{1}} \sum_{m, n=\infty}^{\infty} \tilde{u}(m, n) \exp i\left(m b_{1} x+n b_{2} y\right) \\
& v^{\prime}=\exp i k_{z} z \frac{1}{b_{2}} \sum_{m, n=\infty}^{\infty} \tilde{v}(m, n) \exp i\left(m b_{1} x+n b_{2} y\right) \\
& w^{\prime}=\exp i k_{z} z \sum_{m, n=\infty}^{\infty} \tilde{w}(m, n) \exp i\left(m b_{1} x+n b_{2} y\right)
\end{aligned}
$$

with a similarly defined pressure variable. (Note that we have suppressed the time dependence in $\tilde{u}, \tilde{v}$ and $\tilde{w}$.) Upon equating coefficients of $\exp i\left(k_{z} z+m b_{1} x+n b_{2} y\right)$ and using the continuity equation to eliminate the pressure, we get the following equations:

$$
\begin{aligned}
& \frac{\partial \tilde{u}(m, n)}{\partial t}=\frac{b_{1} b_{2} R_{x}(m, n)}{b_{1}^{2}+b_{2}^{2}}-\nu\left(m^{2} b_{1}^{2}+n^{2} b_{2}^{2}+k_{z}^{2}\right) \tilde{u}(m, n) \\
& \frac{\partial \tilde{v}(m, n)}{\partial t}=\frac{b_{1} b_{2} R_{y}(m, n)}{b_{1}^{2}+b_{2}^{2}}-\nu\left(m^{2} b_{1}^{2}+n^{2} b_{2}^{2}+k_{z}^{2}\right) \tilde{v}(m, n)
\end{aligned}
$$

where

$$
\begin{aligned}
& R_{x}(m, n)=F(m, n)-\frac{m b_{1}^{2}\left(m F+n G+k_{z} H\right)}{m^{2} b_{1}^{2}+n^{2} b_{2}^{2}+k_{z}^{2}} \\
& R_{y}(m, n)=G(m, n)-\frac{n b_{2}^{2}\left(m F+n G+k_{z} H\right)}{m^{2} b_{1}^{2}+n^{2} b_{2}^{2}+k_{z}^{2}}
\end{aligned}
$$

and

$$
\begin{gathered}
F(m, n)=-\frac{m}{2}[\tilde{u}(m-1, n-1)+\tilde{u}(m-1, n+1)-\tilde{u}(m+1, n-1)-\tilde{u}(m+1, n+1)] \\
+\frac{n}{4}[\tilde{u}(m-1, n-1)-\tilde{u}(m-1, n+1)+\tilde{u}(m+1, n-1)-\tilde{u}(m+1, n+1)] \\
-\frac{n}{4}[\tilde{v}(m-1, n-1)+\tilde{v}(m-1, n+1)-\tilde{v}(m+1, n-1)-\tilde{v}(m+1, n+1)]
\end{gathered}
$$




$$
\begin{aligned}
& -\frac{k_{z}}{4}[\tilde{w}(m-1, n-1)+\tilde{w}(m-1, n+1)-\tilde{w}(m+1, n-1)-\tilde{w}(m+1, n+1)](11) \\
& G(m, n)=-\frac{m}{4}[\tilde{v}(m-1, n-1)+\tilde{v}(m-1, n+1)-\tilde{v}(m+1, n-1)-\tilde{v}(m+1, n+1)] \\
& +\frac{n}{2}[\tilde{v}(m-1, n-1)-\tilde{v}(m-1, n+1)+\tilde{v}(m+1, n-1)-\tilde{v}(m+1, n+1)] \\
& +\frac{m}{4}[\tilde{u}(m-1, n-1)-\tilde{u}(m-1, n+1)+\tilde{u}(m+1, n-1)-\tilde{u}(m+1, n+1)] \\
& +\frac{k_{z}}{4}[\tilde{w}(m-1, n-1)-\tilde{w}(m-1, n+1)+\tilde{w}(m+1, n-1)-\tilde{w}(m+1, n+1)]
\end{aligned}
$$

$$
\begin{aligned}
& H(m, n)=-\frac{m}{4}[\tilde{w}(m-1, n-1)+\tilde{w}(m-1, n+1)-\tilde{w}(m+1, n-1)-\tilde{w}(m+1, n+1)] \\
& +\frac{n}{4}[\tilde{w}(m-1, n-1)-\tilde{w}(m-1, n+1)+\tilde{w}(m+1, n-1)-\tilde{w}(m+1, n+1)]
\end{aligned}
$$

and, everywhere, $\tilde{w}(m, n)$ is given by

$$
\tilde{w}(m, n)=-\frac{m \tilde{u}(m, n)+n \tilde{v}(m, n)}{k_{z}}
$$

Since $\tilde{w}$ can be eliminated from $R_{x}$ and $R_{y}$ by means of the last equation, Eqs. (7) and (8) are a system of linear equations for $\tilde{u}(m, n)$ and $\tilde{v}(m, n)$ for $-\infty<m, n<\infty$.

The conditions that the bounding box be impenetrable requires that $u^{\prime}$ be zero for $x=0$ and $L_{1}$ for all $y$ and $z$, and $v^{\prime}$ be zero for $y=0$ and $L_{2}$ for all $x$ and $z$. A consistent set of symmetry conditions which ensure these constraints are the following, for all integer values of $m, n$,

$$
\begin{gathered}
\tilde{u}(-m, n)=-\tilde{u}(m, n) \\
\tilde{v}(-m, n)=\tilde{v}(m, n) \\
\tilde{w}(-m, n)=\tilde{w}(m, n) \\
\tilde{u}(m,-n)=\tilde{u}(m, n) \\
\tilde{v}(m,-n)=-\tilde{v}(m, n) \\
\tilde{w}(m,-n)=\tilde{w}(m, n) .
\end{gathered}
$$

It follows from these that $\tilde{u}(0, n)=\tilde{v}(m, 0)=0$ and that $\tilde{u}(0,0)=\tilde{v}(0,0)=0$. It can be shown that these conditions persist if they are satisfied by the initial conditions. These conditions mean that Eqs. (7) and (8) only have to be solved on the first quadrant of the $m, n$ plane, i.e., $m=0,1,2, \ldots, N, n=0,1,2, \ldots, N$, 
where $N$ is a large integer where we truncate the system. For instance, if we write Eq. (7) with $m=0$ and some $n$, this would require $F(0, n)$, and from Eq. (11) we see that we would need $\tilde{u}(-1, n-1)$. From Eq. (15), this is equal to $-\tilde{u}(1, n-1)$, which is within the solution set. In general, the column $m=-1$ and the row $n=-1$ are shifted into the solution set.

From the structure of the equations, one can see that an equation for mode $(m, n)$ is only coupled to four neighboring modes $(m-1, n-1),(m-1, n+1),(m+1, n-1)$, and $(m+1, n+1)$. These are the nearest four on diagonal lines through $(m, n)$, and these four are each coupled to the nearest four on its diagonals, and so on. This means that the modes can be decomposed into two independent "checkerboard" subsets, the "even" modes where $m+n$ is an even integer and the "odd" modes where $m+n$ is an odd integer. This was pointed out by Bayly (1989).

In the numerical work, we have taken $E=2$ and set the length and time scales by taking the maximum vorticity of the basic flow to be unity $(A=1)$ and $L_{1}=5.6$, $L_{2}=2.8$. The Reynolds number is $\nu^{-1}$ in this dimensionless scheme. One could, of course, rescale the box so that one of the sides has unit length and rescale the Reynolds number and wavenumbers accordingly. The reason for our particular choice was determined by the requirements of the nonlinear code which will be described in the next section.

Equations (7) and (8) were solved by a second order Runge-Kutta method over a range of values of the parameters $R_{e}$ and $k_{z}$. Even or odd modes were excited by taking initial conditions in which all the mode amplitudes were zero except $\tilde{u}(2,0)=1$ to generate even modes or $\tilde{u}(1,0)=1$ for odd modes. Energy growth rates $\beta$ were computed from

$$
\beta=\lim _{t \rightarrow \infty} \frac{\ln (\text { energy })}{t}
$$

where "energy" is the sum of the squares of all the mode amplitudes. It was necessary to integrate for a long time to approach an asymptote in this formula. Over the range computed, we have found that the cutoff $N=40$ was adequate. The results are presented in two figures. Figure 2 shows the neutral curve $(\beta=0)$ for both even and odd modes up to Reynolds numbers of 2000. The band of unstable wavenumbers rapidly expands with increasing Reynolds number. Figure 3 shows the growth rate $\beta$ versus wavenumber $k_{z}$ at Reynolds number 2000. Computations at Reynolds numbers 200,500 , and 1000 are similar, with a pronounced notch in the odd mode curve. The growth rate curves computed by Robinson and Saffman (1984) for the strained vortex have a similar shape made up of the union of separate growth rate curves for modes with differing internal structure, which look like inverted parabolas. The growth rate at their second peak (multiplying their result by 2 to get energy growth rate) is about .3 for $E=2$, which is comparable to our value of about .24 .

A result for unstable plane waves in the unbounded flow with elliptical streamlines may be obtained from Landman and Saffman (1987). The result is

$$
\begin{array}{r}
\frac{\beta}{\omega_{0}}=2\left(\frac{\sigma_{0}}{\omega_{0}}-\nu K^{-2}\right) \\
\sigma_{-} 4
\end{array}
$$

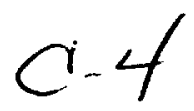




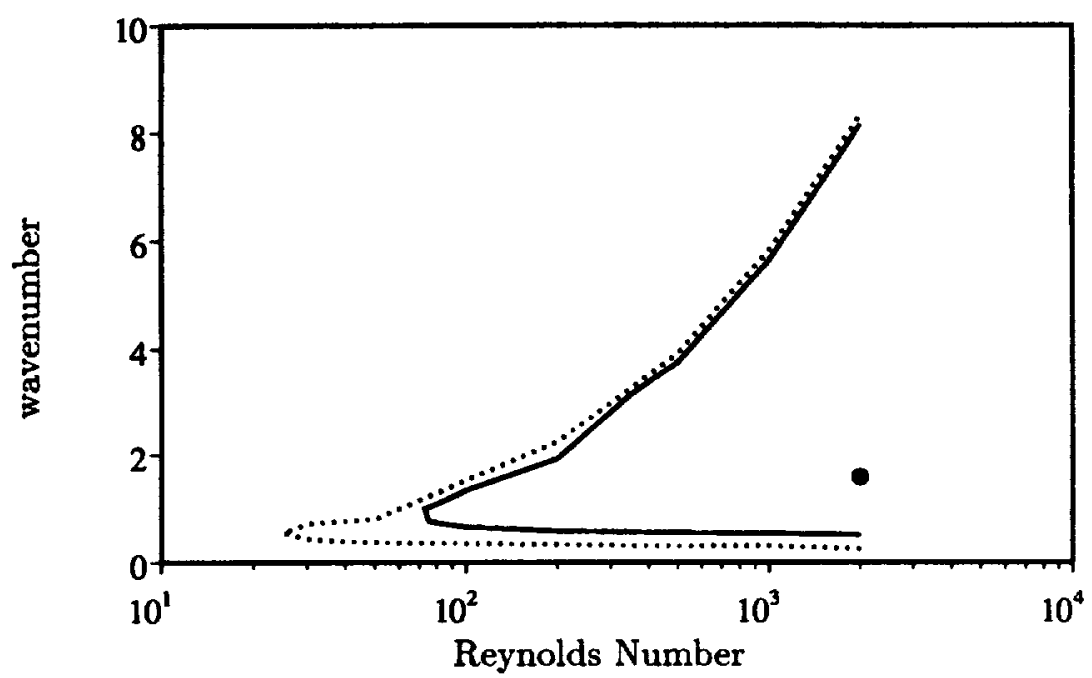

FiguRE 2. Neutral stability curves, $E=2$ : even modes; odd modes. Heavy dot is at $R_{e}$ number and lowest wave number used in the computation.

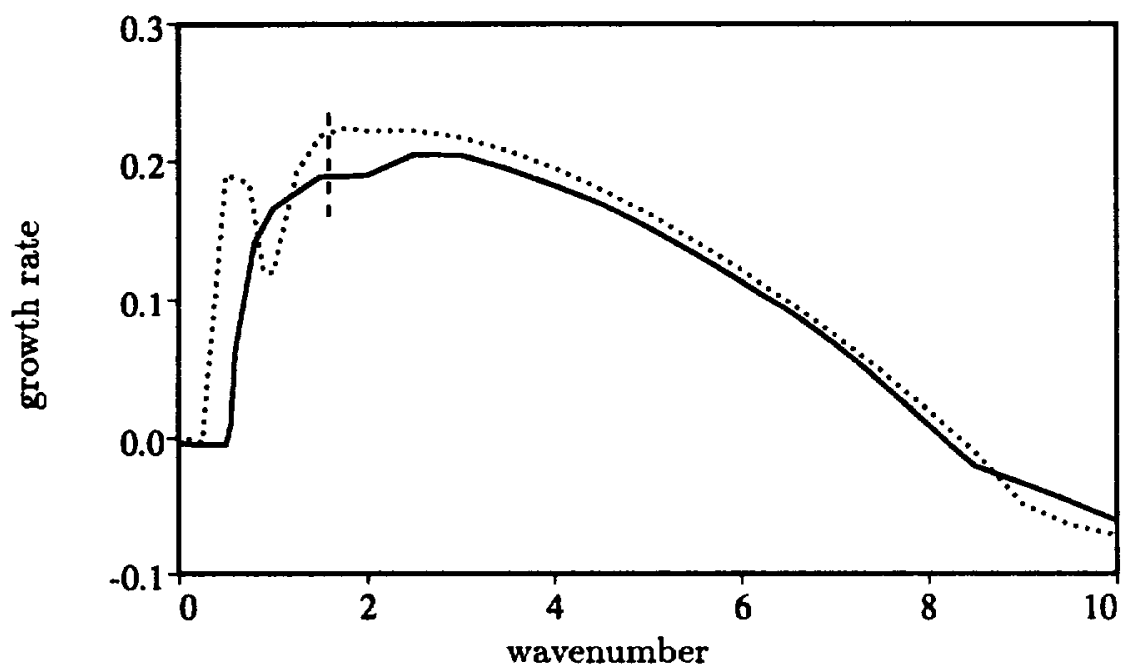

Figure 3. Growth rate versus axial wavenumber at $R_{e}=2000, E=2$ : - even modes; ........ odd modes. Vertical dash is at the lowest wave number used in the computation.

where $\omega_{0}$ is the uniform vorticity, $\sigma_{0}$ is the inviscid amplitude growth rate, and $K^{2}=k_{x}^{2}+k_{y}^{2}+k_{z}^{2}$. The factor two is inserted to convert to energy growth rate. Now $\sigma_{0} / \omega_{0}$ is a function of $\epsilon / \gamma /\left[=\left(E^{2}-1\right) /\left(E^{2}+1\right)\right]$ where $\epsilon$ is strain rate and $\gamma$ is $\omega_{0} / 2$. For $\epsilon / \gamma<.7(E<2.4)$, it is approximately

$$
\frac{\sigma_{0}}{\omega_{0}}=\frac{1}{2}\left(\frac{9}{16}\left(\frac{\epsilon}{\gamma}\right)-.1\left(\frac{\epsilon}{\gamma}\right)^{2}\right) .
$$


For an unstable wave with maximum growth rate, the wavevector makes an angle of $60^{\circ}$ with the rotation axis; therefore, $k_{x}^{2}+k_{y}^{2}=3 k_{z}^{2}$. Using this and $\nu=1 / R_{e}$ gives

$$
\frac{\beta}{\omega_{0}}=2\left(\frac{\sigma_{0}}{\omega_{0}}-\frac{4 k_{z}^{2}}{R_{e}}\right)
$$

For our case with $E=2$ and $\omega_{0}=1$, we get

$$
\beta=.3-\frac{8 k_{z}^{2}}{R_{e}} .
$$

For fixed $R_{e}$, this gives a curve similar in shape to figure 2 with a maximum of .3 at $k_{z}=0$. A neutral curve may be derived from $\mathrm{Eq}$. (24), namely

$$
k_{z}=.19 R_{e}^{1 / 2} .
$$

We can check a few values. At $R_{e}=2000,1000,500$, and 200 the formula gives $8.50,6.01,4.25$, and 2.68, which compare with the computed values 8.32, 5.81, 3.92, and 2.25. The agreement is quite good at the larger Reynolds numbers. Equations (24) and (25) should allow a reasonable extrapolation to Reynolds numbers larger than those for which we have computed.

We conclude that the stability results are similar to those for bending waves on a concentrated vortex at small wavenumbers and similar to results for an unbounded flow with elliptical streamlines at large wavenumber, with comparable growth rates.

\section{Transition to turbulence}

Nonlinear computations have been performed on the Intel Hypercube (i860) using a version of Rogallo's (1981) box code (the "pencil" code) written by Rogallo for this parallel processing machine. This code was designed to solve the Navier-Stokes equations in a box with periodic boundary conditions. The box is not required to have equal sides, but must have volume $(2 \pi)^{3}$. We will take the sides to be $2 L_{1} \times 2 L_{2} \times L_{3}$ with the $x$ and $y$ dimensions double the size of the impenetrable box. By using proper symmetry in the initial conditions, we can insure that there is no flow across the surfaces $x=0, L_{1}$ and $y=0, L_{2}$. The finite dimension in the $z$ direction means that $L_{3}$ is the longest axial wavelength allowed. Defining $b_{1}=\pi / L_{1}, b_{2}=\pi / L_{2}$ as before and $b_{3}=2 \pi / L_{3}$, the volume constraint makes $b_{1} b_{2} b_{3}=1$. The parameter $b_{3}$ is the smallest non-zero axial wavenumber. Allowed values are $k_{z}=b_{3} k$, where $\mathrm{k}$ is an integer. Solutions in this periodic system are thus of the form

$$
\begin{aligned}
& u(x, y, z, t)=\sum_{m, n, k=-N}^{N} \tilde{u}(m, n, k) \exp i\left(m b_{1} x+n b_{2} y+k b_{3} z\right) \\
& v(x, y, z, t)=\sum_{m, n, k=-N}^{N} \tilde{v}(m, n, k) \exp i\left(m b_{1} x+n b_{2} y+k b_{3} z\right)
\end{aligned}
$$




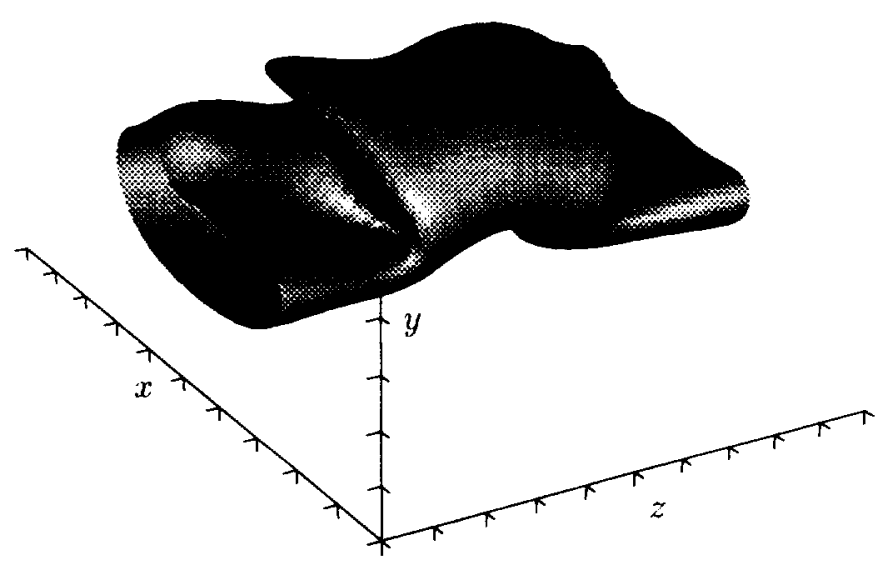

FIGURE 4. Surface of $3 / 8 \max$ vorticity at $T=55.66$. Max vorticity is 2.01 . The tick marks are at .6 intervals in the $x$-direction and .4 intervals in the $y$-and $z$-direction.

$$
w(x, y, z, t)=\sum_{m, n, k=-N}^{N} \tilde{w}(m, n, k) \exp i\left(m b_{1} x+n b_{2} y+k b_{3} z\right)
$$

Conjugate symmetry $\left(\tilde{u}(-m,-n,-k)=\tilde{u}(m, n, k)^{*}\right.$, etc. $)$ is imposed to ensure that the velocity components are real. Symmetries in the initial conditions like those in Eqs. (15)-(19) for each $k$ ensure impenetrability of the side walls. For instance, the velocity field of the basic flow, $U=b_{2} \sin b_{1} x \cos b_{2} y /\left(b_{1}^{2}+b_{2}^{2}\right), V=$ $-b_{1} \cos b_{1} x \sin b_{2} y /\left(b_{1}^{2}+b_{2}^{2}\right)$ is generated by the four modes

$$
\begin{gathered}
\tilde{u}(1,1,0)=\tilde{u}(1,-1,0)=-\tilde{u}(-1,1,0)=-\tilde{u}(-1,-1,0)=-\frac{b_{2} i}{4\left(b_{1}^{2}+b_{2}^{2}\right)} \\
\tilde{v}(1,1,0)=-\tilde{v}(1,-1,0)=-\tilde{v}(-1,1,0)=\tilde{v}(-1,-1,0)=-\frac{b_{2} i}{4\left(b_{2}^{2}+b_{2}^{2}\right)}
\end{gathered}
$$

which have all these symmetries. Perturbations from this can be generated in many different ways and will generate different flows. For instance, we could use random small initial perturbations. For the flow computed here, we excited both even and odd modes by

$$
u^{\prime}=.001 \sin b_{1} x \cos b_{3} k z+.001 \sin 2 b_{1} x \cos b_{3} k z
$$

with $v^{\prime}=0$ and the corresponding $w^{\prime}$ determined from continuity, that is,

$$
\tilde{u}(1,0, k)=-\tilde{u}(-1,0, k)=\tilde{u}(1,0,-k)=-\tilde{u}(-1,0,-k)=-.001 i / 4
$$




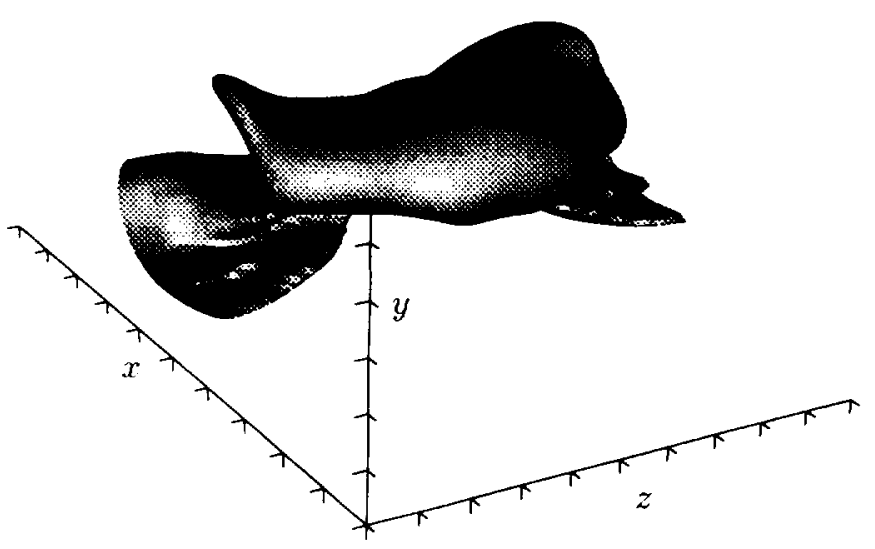

FIGURE 5. Surface of $3 / 8$ max vorticity at $T=61.71$. Max vorticity is 2.78 . See Figure 4 for the value of the intervals on the axes.

$$
\tilde{u}(2,0, k)=-\tilde{u}(-2,0, k)=\tilde{u}(2,0,-k)=-\tilde{u}(-2,0,-k)=-.001 i / 4
$$

for all integer $k$. With the cosine dependence in the $z$ direction, the endwalls and the midplane $z=L_{3} / 2$ will also be impenetrable.

Finally, we chose to do the computation with $E=2=L_{1} / L_{2}$ and $L_{3}=\sqrt{L_{1} L_{2}}$. The volume constraint then makes $L_{1}=5.60, L_{2}=2.8, L_{3}=3.96$, and then $b_{1}=$ $.56, b_{2}=1.12$, and $b_{3}=1.59$. We have computed with $R_{e}=2000$ and $N=128$. The lowest wave number in the initial conditions, $k_{z}=1.59$, is shown in the stability diagrams as a circle in figure 2 and as a vertical dashed line in figure 3 . It is near the position of maximum growth rate and was chosen for this reason.

The major results of the computations are presented in figures 4-9, where surfaces of constant magnitude of the vorticity are shown at 6 different times during the evolution of this flow. In each figure, the surface is of the vorticity value which is $3 / 8$ of the maximum vorticity at this time. Both the time and the value of the maximum vorticity are given in the figure legends. The coordinate axes shown have $x$ to the left, $y$ vertical, and $z$ off to the right. The viewpoint and illumination are from the origin. At the initial time (not shown), the surface is approximately an elliptic cylinder. The instability causes distortion into a wave oriented roughly along a $45^{\circ}$ plane with the vortex moving downward and toward positive $x$ at the ends of the box and upward and toward negative $x$ in the middle. The secondary flow which causes this motion also causes the vortex to be greatly distorted into sheet-like structures which resemble the "cups" found by Rogers and Moser (1992) in their study of the development of three-dimensional structure in a mixing layer after the primary rollup into spanwise rollers. In figures $10 \mathrm{a}$ and $10 \mathrm{~b}$, we look 


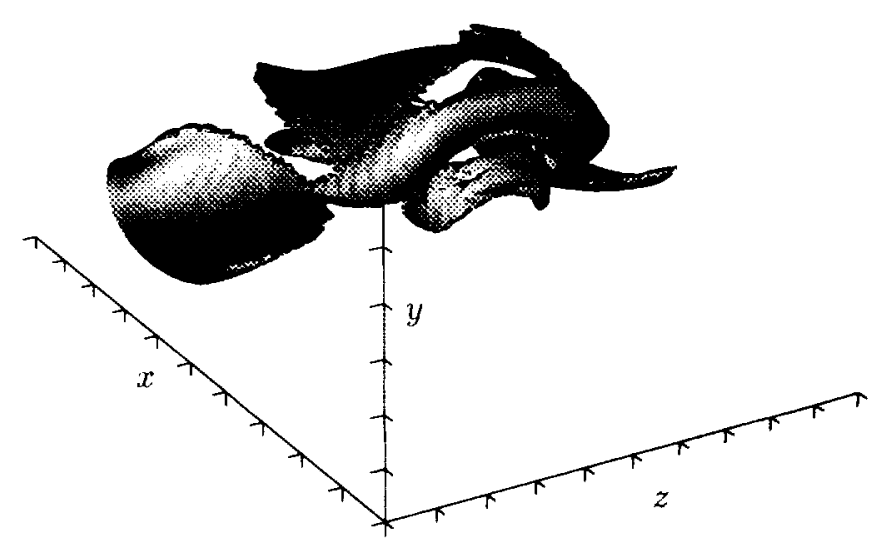

Figure 6. Surface of $3 / 8 \max$ vorticity at $T=70.43$. Max vorticity is 5.50. See Figure 4 for the value of the intervals on the axes.

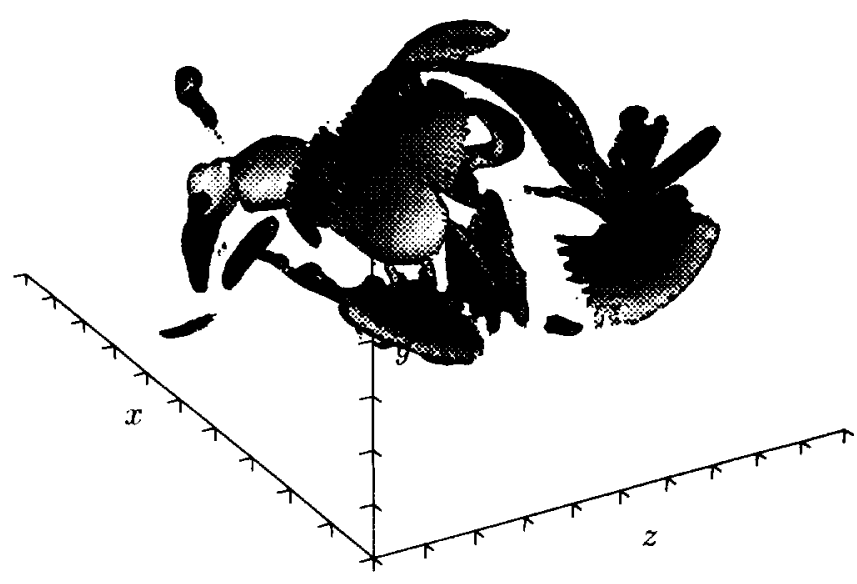

FIGURE 7. Surface of $3 / 8 \max$ vorticity at $T=79.09$. Max vorticity is 9.61 . See Figure 4 for the value of the intervals on the axes. 


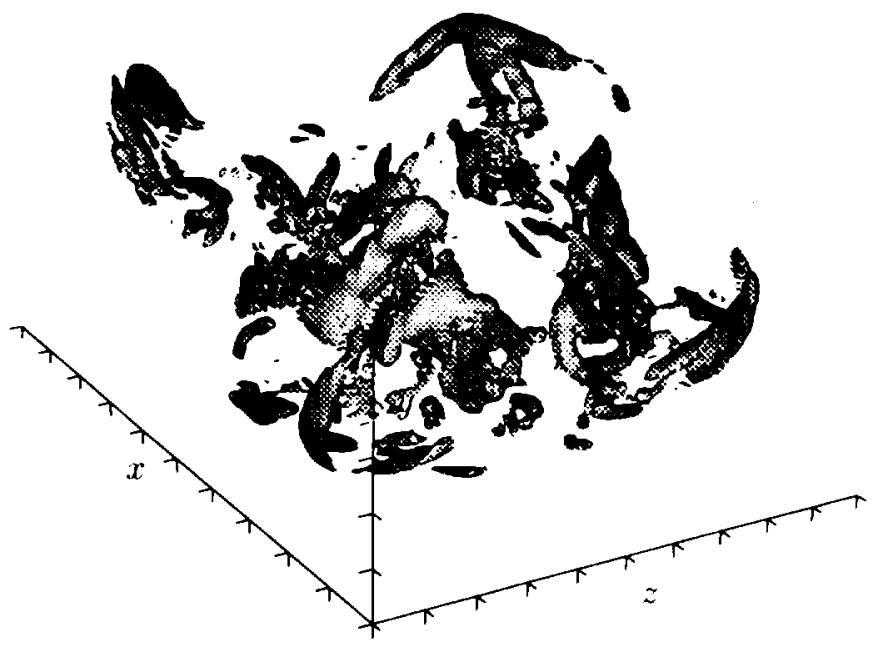

FIGURE 8. Surface of $3 / 8 \max$ vorticity at $T=83.94$. Max vorticity is 13.19 . See Figure 4 for the value of the intervals on the axes.

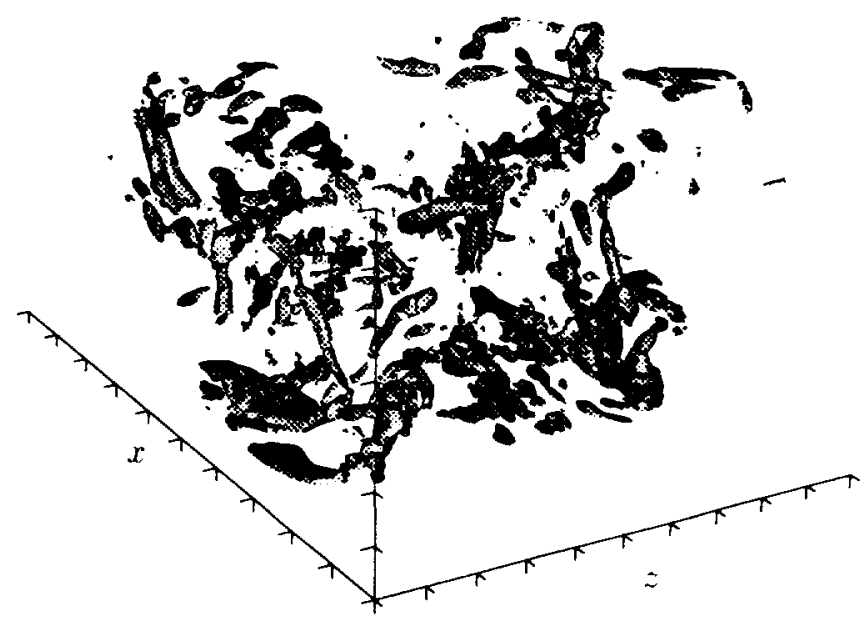

FIgure 9 . Surface of $3 / 8$ max vorticity at $T=87.95$. Max vorticity is 15.70 . See Figure 4 for the value of the intervals on the axes. 

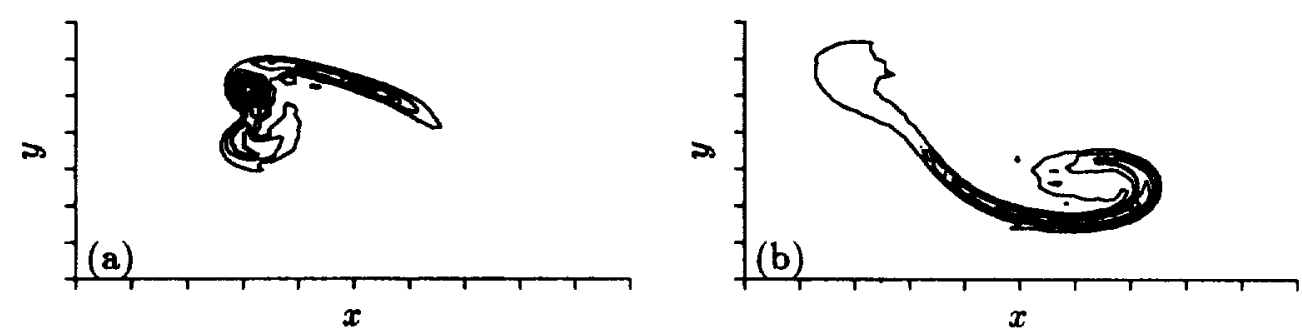

FigURE 10. Vorticity contours at the time corresponding to figure 6 ; a) on the plane $z=L_{3} / 2 ; \mathrm{b}$ ) on the planes $z=0$ and $z=L_{3}$. See Figure 1 for the value of the intervals on the axes.

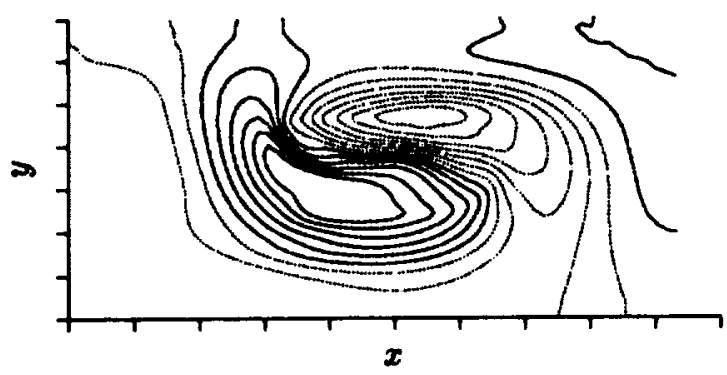

Figure 11. Contours of axial velocity on the plane $z=L_{3} / 4$ at the time corresponding to figure 6 . Solid contours are positive velocity, dotted contours are negative velocity. Maximum velocities are \pm .35 . See Figure 1 for the value of the intervals on the axes.

at vorticity contours on cuts through the structure shown in figure 6 in order to elucidate its structure. In figure 10a, we have taken the cut through the middle of the structure along the plane $z=L_{3} / 2$. By symmetry only, the $z$ component of vorticity is nonzero on this plane. The maximum vorticity in the box, 5.5 , occurs at the center of the rather strong round vortex which is apparent here. In the three-dimensional rendering this vortex is seen from in front and appears to be like a horseshoe vortex. The heavier contour line in figure $10 \mathrm{a}$ is at the same vorticity level as the surface in the three-dimensional view. In figure $10 \mathrm{~b}$, the cut is at the ends of the box at planes $z=0$ and $z=L_{3}$, where again only $\omega_{z}$ is non-zero. The maximum vorticity in the strong sheet-like structure is 3.5 .

In figure 11, we show contours of the axial velocity $w$ in the plane $z=L_{3} / 4$ through the same structure shown in figure 6 . This is more regular than we expected and shows characteristics of the 1,1 mode in Robinson and Saffman (1984). The first 1 refers to the angular wave number and the second to the number of nodes in the axial velocity. Here, despite the already complicated vorticity, the underlying secondary flow of the instability is still evident, much amplified, with maximum velocities \pm .35 . Since $w$ is zero at both ends of the box and in the middle, and $w$ on the plane $z=3 L_{3} / 4$ is just the reverse of that shown in figure 11 , we can picture the secondary flow as two eddies, one in each half of the box, with the velocity toward 


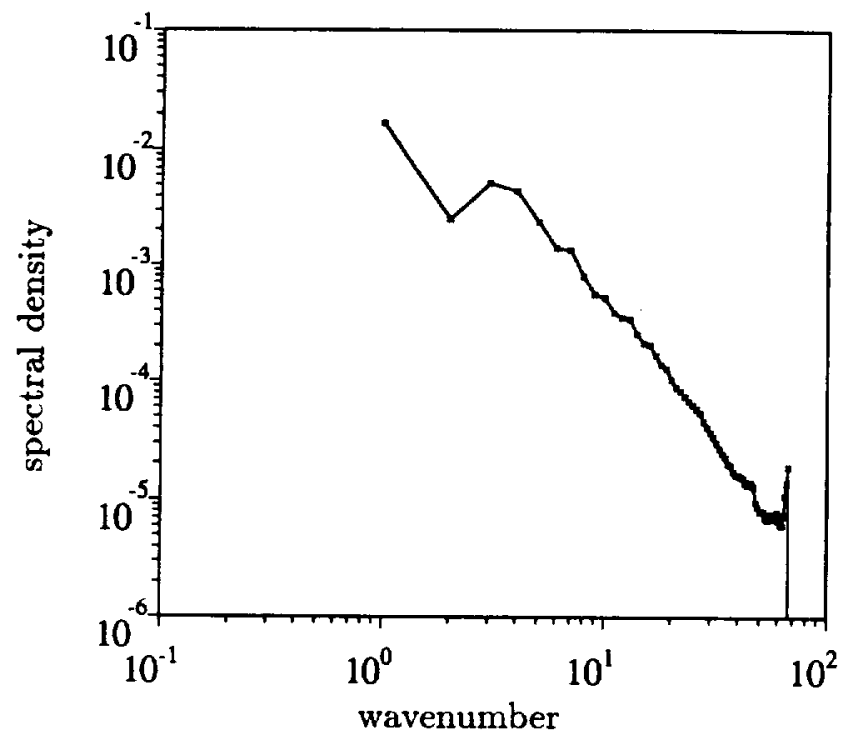

Figure 12. Energy spectrum at $T=87.95$.

the center of the box in the lower part and away from the center above. Completion of this circulation is such as to smash the vorticity downwards and toward positive $x$ at the ends while stretching it, and upward and toward negative $x$ in the middle, stretching it here also. What is remarkable is that this secondary motion is evident throughout the entire sequence of views, even at the time of figure 9 where it is quite irregular but with the main trends described above.

As we proceed from figure 6 to figure 7 , the strong vortex which was evident in figure 6 has disappeared. The central part has apparently been carried downward into the compressive part of the circulation which decreased its vorticity below the plotting level. The remnants of its sides have been stretched into the shield-shaped vortex sheets on each side of the center. In the next view, figure 8 , there is no vorticity at the level plotted across the plane $z=L_{3} / 2$. This is not clear from the figure, but when we rotated it we had an open view down the middle. The structures we see are mostly tubelike now, and this is even more evident in figure 9 , which is the time at which the maximum vorticity in the box is largest. At later times, this begins to decrease, but the tubelike nature of the vorticity is evident still at the time of 103.8 when we ended the computation.

Figure 12 shows the three dimensional energy spectrum at the time of figure 9. While this is quite broad, indicating turbulence, we do not see $a-5 / 3$ range at this Reynolds number.

\section{Discussion}

Our overall impression of this flow is of two large counter-rotating turbulent eddies, each carrying many intense interacting vortex tubes. One can see the development of this pretty clearly in figures 7 and 8 , less clearly in figure 9 . This motion was already present as the growing secondary flow of the instability and 


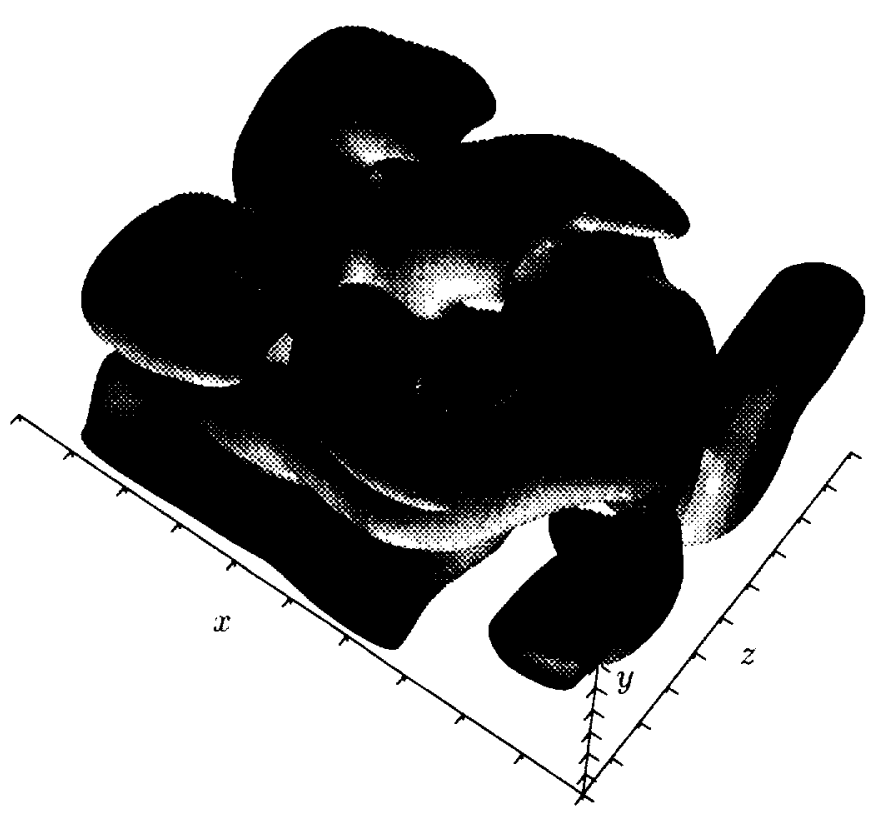

FIGURE 13. Surface of $3 / 8 \max$ filtered vorticity at $T=87.95$. Max filtered vorticity is 1.19 . See Figure 4 for the value of the intervals on the axes.

was the controlling factor in the development of the vortex sheets. To understand the large scale swirling motion without the masking effect of the small vortices, we have filtered out the high wavenumber part of the velocity with a Gaussian filter with width .2 . We have computed the magnitude of the vorticity in this filtered flow at the same time as in figure 9. The result is presented in figure 13 as a surface of $3 / 8$ of the maximum vorticity, which is 1.19 in this filtered flow. This looks like a horseshoe vortex with two vortices wrapping around each of its counterrotating legs. The head of the vortex, which is lifted up, contains the point of maximum vorticity. The sense of motion is upward between the legs of the vortex and forward over the top of the nose, which is the sense of rotation of the original swirling flow.

The evolution of the sheets into tubes appears to be through the Kelvin-Helmhotz instability, or by the Lin-Corcos (1984) version which includes vortex stretching, but nothing very dramatic occurs. Local thick spots gradually become thicker and develop into tubes. In some preliminary computations at higher Reynolds number, the cup-shaped vortex sheet developed into three clearly defined parallel vortex tubes.

In the future, we plan to compute this flow at higher Reynolds number and greater resolution with the objective of looking more carefully at the development of small scale structure. As a generator of small scale turbulence, this flow is similar to the Taylor-Green flow (Brachet et al., 1983). The present flow has some advantages for the study of turbulence because of its development from a typical instability and the fact that the turbulent region tends to avoid close contact with the impenetrable boundaries. 


\section{Acknowledgement}

The authors would like to acknowledge several very useful discussions with $\mathrm{Dr}$. Karim Shariff of NASA-Ames. We are especially grateful to Dr. Robert Rogallo for allowing us to use his computer program and to Dr. Michael Rogers for generously providing us with his data reduction code. The direct numerical simulations were performed on the iPSC/860 at the Numerical Aerodynamic Simulation facility, NASA Ames Research Center.

\section{REFERENCES}

BAYLY, B. J. 1986 Three-Dimensional Instability of Elliptical Flow. Phys. Rev. Lett. 57, 2160-2163.

BAYLY, B. J. 1989 Computations of Broad-Band Instabilities in a Class of ClosedStreamline Flows. In Mathematical Aspects of Vortex Dynamics, (ed. R. E. Caflisch.) SIAM, Philadelphia, PA, 50-58.

Brachet, M. E., Meiron, D. I, Orszag, S. A., Nickel, B. G., Morf, R. H. \& FRISCH, U. Small-scale structure of the Taylor-Green vortex J. Fluid Mech. 190. 411-452.

LIN, S. J. \& Concos, G. M. 1984 The mixing layer: deterministic models of a turbulent flow. Part 3. The effect of plane strain on the dynamics of streamwise vortices. J. Fluid Mech. 141, 139-178.

Gledzer, Ye. B., Novikov, Yu. V., Obukhov, A. M. \& Chusov, A. M. 1974 An investigation of the stability of liquid flows in a three-axis ellipsoid. Izv., Atmospheric and Oceanic Physics. 10, 115-118.

Gledzer, Ye. B., Dolzhanskiy, F. V., Obukhov, A. M. \& Ponomarev, V. M. 1975 An experimental and theoretical study of the stability of motion of a liquid in an elliptic cylinder. Izv., Atmospheric and Oceanic Physics. 11, 981-992.

LANDMAn, M. J. \& Saffman, P. G. 1987 The three-dimensional instability of strained vortices in a viscous fluid. Phys. Fluids. 30, 2339-2342.

Malkus, W. V. R. 1989 An Experimental Study of Global Instabilities Due to the Tidal (Elliptical) Distortion of a Rotating Elastic Cylinder. Geophys. Astrophys. Fluid Dyn. 48, 123-134.

OrszaG, S. A. \& Patera, A. T. 1983 Secondary instability of wall-bounded shear flows. J. Fluid Mech. 128, 347-385.

Pierrehumbert, R. T. 1986 Universal Short-Wave Instability of Two Dimensional Eddies in an Inviscid fluid. Phys. Rev. Lett. 57, 2157-2159.

Rogallo, R. S. 1981 Numerical Experiments in Homogeneous Turbulence. NASA Technical Memorandum 81315.

Rogers, M. M. \& Moser, R. D. 1992 The three-dimensional evolution of a plane mixing layer: the Kelvin-Helmholtz rollup. J. Fluid Mech. 243, 183-226. 
Waleffe, F. 1989 The 3D Instability of a Strained Vortex and its Relation to Turbulence. Ph.D Thesis, Massachusetts Institute of Technology.

WALEFFE, F. 1990 On the three-dimensional instability of strained vortices. Phys. Fluids A. 2, 76-80. 